\title{
Kernel-Based Manifold Learning for Statistical Analysis of Diffusion Tensor Images
}

\author{
Parmeshwar Khurd, Ragini Verma, and Christos Davatzikos \\ Section of Biomedical Image Analysis, Dept. of Radiology, \\ University of Pennsylvania, Philadelphia, USA \\ khurdp@uphs . upenn. edu
}

\begin{abstract}
Diffusion tensor imaging (DTI) is an important modality to study white matter structure in brain images and voxel-based group-wise statistical analysis of DTI is an integral component in most biomedical applications of DTI. Voxel-based DTI analysis should ideally satisfy two desiderata: (1) it should obtain a good characterization of the statistical distribution of the tensors under consideration at a given voxel, which typically lie on a non-linear submanifold of $\Re^{6}$, and (2) it should find an optimal way to identify statistical differences between two groups of tensor measurements, e.g., as in comparative studies between normal and diseased populations. In this paper, extending previous work on the application of manifold learning techniques to DTI, we shall present a kernel-based approach to voxel-wise statistical analysis of DTI data that satisfies both these desiderata. Using both simulated and real data, we shall show that kernel principal component analysis (kPCA) can effectively learn the probability density of the tensors under consideration and that kernel Fisher discriminant analysis (kFDA) can find good features that can optimally discriminate between groups. We shall also present results from an application of kFDA to a DTI dataset obtained as part of a clinical study of schizophrenia.
\end{abstract}

\section{Introduction}

Diffusion tensor imaging (DTI) has become an important modality for studying white matter structure in brain imaging and other biomedical applications [1]. Several such applications require a group-wise statistical analysis of DT images which can identify regional differences between two groups of DT images. However, statistical analysis of DTI is complicated by the fact that we now have a $3 \times 3$ positive-definite symmetric matrix or diffusion tensor at each voxel instead of a single value as in the case of scalar images.

In conventional methods of performing such an analysis, scalar or vector images are first computed from the DTI dataset and then spatially normalized to a common template. A statistical p-value map is then computed from these scalar or vector images with the application of standard tests for statistical inference. Commonly used scalar images for such analyses include the fractional anisotropy (FA) and the apparent diffusion coefficient (ADC) 11. Non-scalar features [2] such as the principal eigendirections of the tensors have also been used 
in some analyses. However, a main disadvantage of these methods is that they do not use the complete information available in the DTI dataset, but rather make the a priori assumption that group differences will affect specific quantities to be extracted from the diffusion tensor, such as FA. Moreover, differences in these scalar maps may be mutually difficult to interpret.

A voxel-based statistical analysis should ideally try to learn the density and the manifold structure pertinent to the specific tensors under consideration and determine the degree of separation between two groups of tensors. Methods for statistical analysis of tensors have been recently developed that utilize the full tensor information by trying to learn the underlying structure of the data. These methods fall into two categories: methods based upon Riemannian symmetric spaces [34] and methods based upon manifold learning [5]. Methods based upon Riemannian symmetric spaces 34 rely upon the assumption that the tensors around a given voxel from various subjects belong to a principal geodesic (sub)manifold and that these tensors obey a normal distribution on that sub-manifold. The basic principle of these methods is sound, namely that statistical analysis of tensors must be restricted to the appropriate manifold of positive definite symmetric tensors, which is known to be a cone embedded in $\Re^{6}$. However, there is no guarantee that the representations of the tensors on this sub-manifold will have normal distributions, and most importantly, restricting the analysis on the manifold of positive definite symmetric tensors is of little help in hypothesis testing studies, since the tensors measured at a given voxel or neighborhood from a particular set of brains typically lie on a much more restricted submanifold. Our main goal is to determine the statistical distribution of tensors on this submanifold. In the approaches based upon manifold learning [5], the focus was on learning embeddings (or features) parameterizing the underlying manifold structure of the tensors. The learned features belonged to a low-dimensional linear manifold parameterizing the higher-dimensional tensor manifold and were subsequently used for group-wise statistical analysis. The main problem with manifold learning approaches [6] is that although they estimate the embedding of the manifold that represents the tensor measurements fairly well, they fail to estimate the probability distribution (non-Gaussian) on the (flattened) manifold itself. From experiments with simulated data, we have found that such an approach does not completely parameterize the probability density of the tensors under consideration and that the learned features do not always identify the differences between the two groups.

We shall present an integrated kernel-based approach to voxel-based analysis that accurately estimates the underlying distribution of the tensor data, obtains a highly informative linear representation for the tensors and uses this representation to determine statistically optimal ways of separating the tensor data. We shall build upon related approaches in 5. We present our methods in Sec. 2 and the results from various experiments on simulated and real data in Sec. 3.1 and Sec. 3.2. We shall establish that higher-dimensional kernel principal component analysis (kPCA) features are very effective in learning the required probability density of the tensors from a voxel and that kernel Fisher discriminant analysis 
(kFDA) can optimally discriminate between groups of tensors. We shall also apply our methods to a clinical DTI dataset of healthy controls and schizophrenic patients in Sec. 3.3. We conclude with Sec. 4

\section{Methods}

\subsection{Kernel-Based Approach to Group-Wise Voxel-Based DTI Statistical Analysis}

A DT image consists of a $3 \times 3$ positive-definite symmetric matrix or tensor $T$ at each voxel in the image. We may represent this tensor as $T=\sum_{i=1}^{3} \lambda_{i} \mathbf{v}_{i} \mathbf{v}_{i}^{T}$, where $\lambda_{i}>0$ and $\mathbf{v}_{i}$ represent the eigenvalues and eigenvectors of the tensor, respectively. Pathology can bring about subtle changes in eigen values or angular changes in the eigen vectors, that can be partially seen in measures of anisotropy and diffusivity computed from the tensor, such as FA and ADC, although eigen vector changes can only be seen in full tensor color maps. Group-wise voxelbased statistical analysis of DTI data involves normalizing all DT images to a common DTI template using a suitable technique [7/8/9] and then using an appropriate statistical test to infer regional differences between groups based upon the tensors at (or around) a voxel. We form a voxel-based dataset by collecting tensors from each location as samples. Such samples should ideally be formed using the tensors at a voxel from all subjects, in conjunction with the deformation field used to normalize the tensors. In this paper, we focus on the former term, however our approach readily extends to the latter, as well as to neighborhood information from tensors around a voxel under consideration, albeit at the cost of increasing the dimensionality of the measurements and the embedding space. The samples in our voxel-based dataset will have unknown statistical distributions on complex unknown non-linear manifolds. Therefore, it is particularly important to analyze the underlying structure in our dataset and to use this structure to estimate features that can identify group differences.

We have found that kernel-based techniques (kPCA and kFDA) are ideally suited for performing such an analysis. The common idea behind kernel-based techniques is to transform the samples into a higher-dimensional reproducible kernel Hilbert space (RKHS). Using the "kernel trick", any algorithm that can be written in terms of inner products in the RKHS, can be readily formulated in the original space in terms of the kernel. Moreover, hyperplanes in the RKHS, such as the ones spanned by principal eigenvectors or the ones separating two sets of samples, become nonlinear hyper-surfaces in the original space, thereby allowing us to nonlinearly span or separate groups of samples for the purposes of density estimation or classification, respectively. We can hence easily perform various calculations such as computing projections onto directions in the RKHS, although we cannot visualize this space itself. Figure 11illustrates the idea behind obtaining such projections.

From our voxel-based samples, we can obtain highly informative projections using the kPCA technique, as described later in Sec. 2.2. Although we could extract useful features from this higher-dimensional kPCA representation to aid 


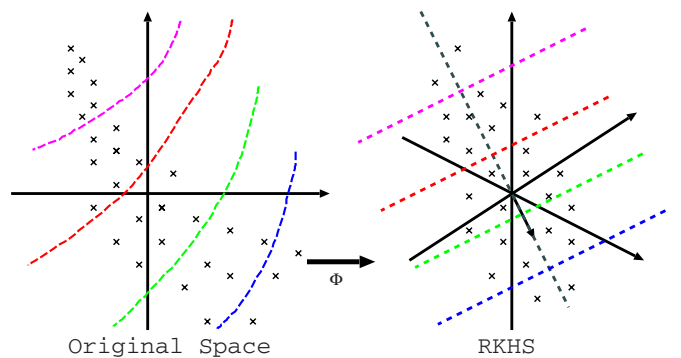

The mapping $\boldsymbol{\Phi}$ takes points (marked with crosses) from the original space to the RKHS. Hyperplanes having constant projections onto a vector in the RKHS become curved lines in the original space. Such curved lines can give us important insight into how the corresponding RKHS projection parameterizes the original points.

Fig. 1. Kernel-based projections

us in inferring differences between groups, it would be better to perform this inference step in an automated and optimal manner. For this purpose, we shall use the kFDA technique described in Sec. 2.3, which finds scalar projections onto a single RKHS direction that can optimally discriminate between groups. Having obtained our kernel-based features, we can then apply standard statistical tests such as the Hotelling $T^{2}$ test in the case of $\mathrm{kPCA}$ or the t-test in the case of the kFDA to obtain a voxel-wise p-value map. We note that the projections found by $\mathrm{kPCA}$ and $\mathrm{kFDA}$ lie along directions in the linear RKHS and hence linear tests for statistical inference can be reliably applied to these projections in order to identify separation between groups. Regions with low p-values indicate the regions with significant differences between the two groups.

Before we present the $\mathrm{kPCA}$ and $\mathrm{kFDA}$ techniques in detail, here is a brief note on our mathematical conventions: We denote vectors by bold-faced lower case letters, e.g. x, and matrices by upper-case letters, e.g. A. We use e to denote the vector of all 1's and $I$ to denote the identity matrix. We occasionally use $\mathbf{e}_{m}$ to denote a vector of all 1's in $m$-dimensional space. We use the superscripts ${ }^{T}$ and ${ }^{-1}$ to denote the matrix transpose and the matrix inverse respectively. We denote the sample mean of a set of vectors $\left\{\mathbf{x}_{i}, i=1, \cdots, K\right\}$ by $\overline{\mathbf{x}}$. We denote the inner product of two vectors $\mathbf{x}_{i}, \mathbf{x}_{j}$ by $\left\langle\mathbf{x}_{i}, \mathbf{x}_{j}\right\rangle$. We shall assume that our group-wise study involves a statistical analysis of the DT images of $N$ subjects, with $N_{+}$subjects in one class (the positive class) and the remaining $N_{-}$subjects in a second class (the negative class).

\subsection{Kernel Principal Component Analysis (kPCA)}

We now describe the kPCA technique [10] which can find a rich linear representation of our voxel-based samples as well as provide an accurate estimate of the probability density underlying these samples. In conventional PCA, we find out principal directions in the vector space of the samples that maximize the variance of the projections of the samples along those directions and which also minimize the least-squares representation error for the samples. In kPCA, we find similar principal eigendirections in the higher-dimensional RKHS. Let us denote the nonlinear mapping of point $\mathbf{x}$ into the Hilbert space by $\boldsymbol{\Phi}(\mathbf{x})$, and let us denote the underlying kernel by $k(.,$.$) , where \left\langle\boldsymbol{\Phi}\left(\mathbf{x}_{i}\right), \boldsymbol{\Phi}\left(\mathbf{x}_{j}\right)>=k\left(\mathbf{x}_{i}, \mathbf{x}_{j}\right)\right.$. 
Since a principal eigenvector $\mathbf{v}$ in the higher-dimensional Hilbert space lies in the span of the vectors $\boldsymbol{\Phi}\left(\mathbf{x}_{i}\right)-\overline{\mathbf{\Phi}}, i=1, \cdots, N$, it can be conveniently represented as $\mathbf{v}=\sum_{i} \alpha_{i}\left(\boldsymbol{\Phi}\left(\mathbf{x}_{i}\right)-\overline{\boldsymbol{\Phi}}\right)$, where $\boldsymbol{\alpha}$ is an $N$-dimensional vector. Projections of any sample along the eigenvector $\mathbf{v}$ can now be conveniently computed using this new representation in the kernel basis.

The entire kPCA procedure is summarized below [6]:

1. Form the kernel matrix $K$, where $K_{i j}=k\left(\mathbf{x}_{i}, \mathbf{x}_{j}\right), i=1, \cdots, N, j=1, \cdots, N$.

2. Center the kernel matrix to obtain $K_{c}=\left(I-\frac{1}{N} \mathbf{e e}^{T}\right) K\left(I-\frac{1}{N} \mathbf{e e}^{T}\right)$.

3. Eigen-decompose $K_{c}$ to obtain its eigenvectors $\boldsymbol{\alpha}^{(i)}$ and eigenvalues $\lambda_{i}, i=$ $1, \cdots, N$.

4. Normalize the eigenvectors $\boldsymbol{\alpha}^{(i)}$ to have length $\frac{1}{\sqrt{\lambda_{i}}}$ so that the eigenvectors $\mathbf{v}^{(i)}$ in the RKHS have unit length.

5. The $i^{\text {th }} \mathrm{kPCA}$ component for training sample $\mathbf{x}_{k}$ is given by: $<\boldsymbol{\Phi}\left(\mathbf{x}_{k}\right)-\overline{\mathbf{\Phi}}, \mathbf{v}^{(i)}>=\lambda_{i} \alpha_{k}^{(i)}$

6. For a general test point $\mathbf{x}$, the $i^{\text {th }} \mathrm{kPCA}$ component is:

$$
<\boldsymbol{\Phi}(\mathbf{x})-\overline{\mathbf{\Phi}}, \mathbf{v}^{(i)}>=\sum_{m} \alpha_{m}^{(i)} k\left(\mathbf{x}, \mathbf{x}_{m}\right)-\frac{1}{N} \sum_{m, n} \alpha_{m}^{(i)} k\left(\mathbf{x}, \mathbf{x}_{n}\right)
$$

In addition to finding the orthogonal directions of maximal variance in the higher-dimensional RKHS, kPCA also provides an estimate of the probability density underlying the samples. It has been pointed out in 11 that kPCA with a Gaussian radial basis function kernel amounts to orthogonal series density estimation using Hermite polynomials. In Sec. 3.1, we shall present a simulated example (see Fig. 2) where kPCA obtained a very good parameterization of the density underlying the dataset. We also note that the kPCA components constitute a linear representation of the dataset in the RKHS, which considerably simplifies any further analysis such as the application of tests for statistical inference.

\subsection{Kernel Fisher Discriminant Analysis (kFDA)}

As described earlier, often the goal is not only to nonlinearly approximate the probability density from a number of samples, as in Sec. 2.2. but also to nonlinearly separate two groups of samples belonging to separate classes. We now examine this problem using kernel-based discriminants. We describe the kFDA technique 1012, which focuses on finding nonlinear projections of the tensorial data which can optimally discriminate between the two groups. Kernel FDA finds a direction $\mathbf{w}$ in the higher-dimensional RKHS so that a projection along this direction maximizes a separability measure known as the Rayleigh coefficient (or the Fisher discriminant ratio). Let $\overline{\mathbf{\Phi}}^{+}$denote the Hilbert-space mean for the samples in the positive class, corresponding to the $N_{+}$DT images from the first group, and let $\overline{\boldsymbol{\Phi}}^{-}$denote the mean for the negative class, corresponding to the $N_{-}$DT images from the second group. Let $\Sigma^{+}$and $\Sigma^{-}$denote the sample covariance matrices for the positive and negative classes respectively. The Rayleigh coefficient is then given by:

$$
J(\mathbf{w})=\frac{\left(\mathbf{w}^{T}\left(\overline{\boldsymbol{\Phi}}^{+}-\overline{\boldsymbol{\Phi}}^{-}\right)\right)^{2}}{\mathbf{w}^{T}\left(\Sigma^{+}+\Sigma^{-}+c I\right) \mathbf{w}},
$$


where the scalar $c$ is used to introduce regularization. As in $\mathrm{kPCA}$, the optimal solution $\mathbf{w}^{*}$ maximizing $J(\mathbf{w})$ can again be conveniently represented using the samples, i.e. $\mathbf{w}^{*}=\sum_{n} \alpha_{n}^{*} \boldsymbol{\Phi}\left(\mathbf{x}_{n}\right)$, and projections along this direction can be easily obtained. A convenient analytical solution for $\boldsymbol{\alpha}^{*}$ is provided in [12]

$$
\boldsymbol{\alpha}^{*}=\frac{1}{c}\left[I-J(c I+J K J)^{-1} J K\right] \mathbf{a},
$$

where $\mathbf{a}=\mathbf{a}^{+}-\mathbf{a}^{-}, \quad \mathbf{a}^{+}=\left[\begin{array}{c}\frac{1}{N_{+}} \mathbf{e}_{N_{+}} \\ 0\end{array}\right], \quad \mathbf{a}^{-}=\left[\begin{array}{c}0 \\ \frac{1}{N_{-}} \mathbf{e}_{N_{-}}\end{array}\right], \quad J=\left[\begin{array}{cc}J^{+} & 0 \\ 0 & J^{-}\end{array}\right]$,

$$
J^{+}=\frac{1}{\sqrt{N_{+}}}\left(I-\frac{1}{N_{+}} \mathbf{e}_{N_{+}} \mathbf{e}_{N_{+}}^{T}\right), \quad J^{-}=\frac{1}{\sqrt{N_{-}}}\left(I-\frac{1}{N_{-}} \mathbf{e}_{N_{-}} \mathbf{e}_{N_{-}}^{T}\right),
$$

and where $K$ represents the un-centered kernel matrix. An alternative quadratic programming approach to obtaining the solution $\boldsymbol{\alpha}^{*}$ is provided in [10].

To summarize, we have presented the $\mathrm{kPCA}$ technique which can estimate the probability density and yield a rich linear representation for any voxel-based tensor dataset and the kFDA technique which uses this representation to extract features that can identify group differences in a statistically optimal manner.

\section{Results and Discussion}

We have applied the kernelized framework to the group analysis of real and simulated DTI data with the aim of demonstrating that $\mathrm{kPCA}$ is able to capture the statistical distribution underlying such datasets and that application of kFDA facilitates optimal group-based separation.

\subsection{Statistical Analysis of Simulated Tensors}

We shall first apply our kernelized framework to group analysis of simulated (non-imaging) tensor datasets where we know the ground-truth underlying structure in the dataset and can therefore easily validate our results. We consider tensor datasets with changes in the principal eigenvalue as well as in one of the angles describing the principal eigendirection. Such subtle changes emulate pathology-induced changes that affect the eigenvalue and eigenvector and an analysis of subtle changes of this nature may be of particular importance in studying complex brain disorders such as schizophrenia which result in non-focal regional changes. We have found that $\mathrm{kPCA}$ is well-suited for parameterizing the density for such datasets and that kFDA can effectively highlight the differences that discriminate between groups. For ease of visualization, we present representative results on a dataset with variation in the radial and angular directions instead of on a tensorial dataset with changes in the principal eigenvalue and eigendirection.

kPCA representation: Our dataset consisted of points forming a semi-circular band (see Fig. 2(a)) and was generated using 36 angles (in the $0-144$ degrees range) and 6 radial values (in the range 1.3-1.8) resulting in a total of 216 points. 


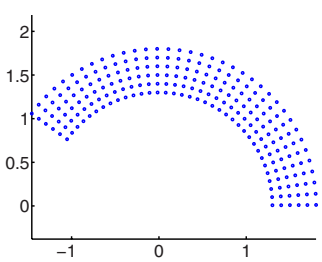

(a)

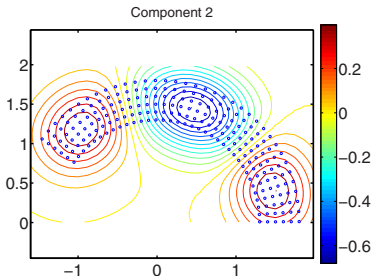

(d)

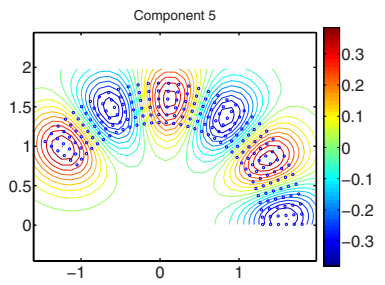

(g)

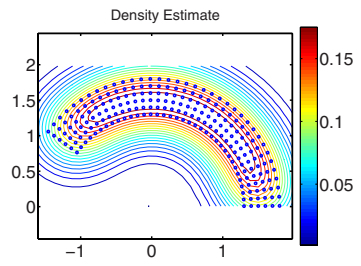

(b)

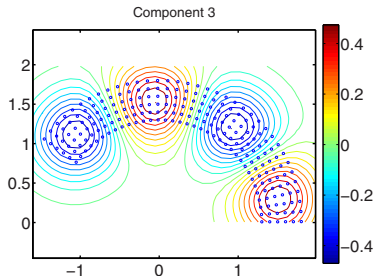

(e)

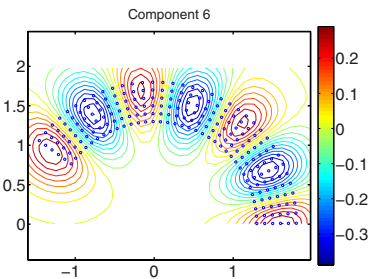

(h)

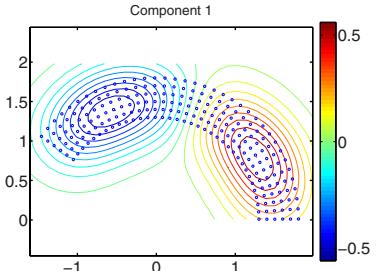

(c)

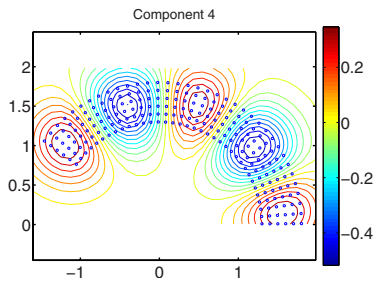

(f)

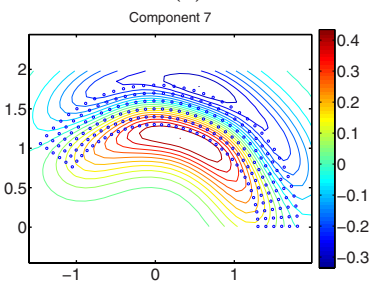

(i)

Fig. 2. (a) Dataset, (b) Contour plot for kernel probability density estimate, (c) Contour plot for $1^{\text {st }} \mathrm{kPCA}$ component, (d) Contour plot for $2^{\text {nd }} \mathrm{kPCA}$ component, (e) Contour plot for $3^{\text {rd }} \mathrm{kPCA}$ component, (f) Contour plot for $4^{\text {th }} \mathrm{kPCA}$ component, (g) Contour plot for $5^{\text {th }} \mathrm{kPCA}$ component, (h) Contour plot for $6^{\text {th }} \mathrm{kPCA}$ component, and (i) Contour plot for $7^{\text {th }} \mathrm{kPCA}$ component (Please see text for explanation)

We applied kPCA to this dataset using a Gaussian radial basis function (RBF) as our kernel with the kernel width $\sigma^{2}$ set to 0.1 . The kernel width parameter was chosen to be a suitable function of the average distance between nearest neighbors $\mathbf{x}_{i}$ and $\mathbf{x}_{j}$, i.e. $\left\|\mathbf{x}_{i}-\mathbf{x}_{j}\right\|$ and the number of samples, and our choice was motivated by the desire to obtain meaningful representations for the different kPCA components as well as a reasonably good probably density estimate for the entire dataset. The iso-contours for our kernel probability density estimate (using all kPCA components), shown in Fig. 2(b), indicate that the estimated density is roughly constant in the central regions where data points are uniformly present and that the density estimate smoothly fades to zero as we approach the data points lying along the borders. The iso-contour plots for 7 principal kPCA components, shown in Fig. 2(c)-(i), represent the hyperplanes having constant projections onto the corresponding 7 RKHS eigen-vectors, as explained earlier in Fig. 11 It can be seen that the first 6 components (Fig. 2(c)-(h)) represent the angular changes in the data using varying scales, e.g., the third kPCA component (Fig. 2(e)) divides the angular variation in the data into four 


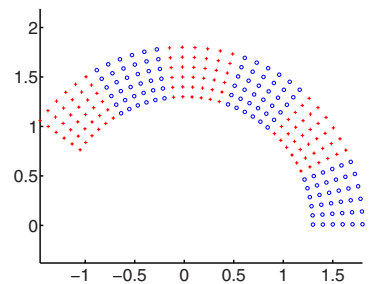

(a)

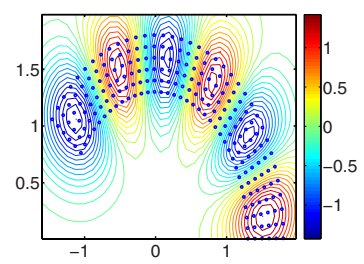

(b)

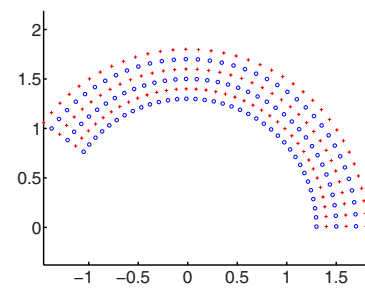

(c)

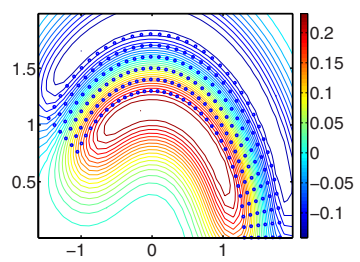

(d)

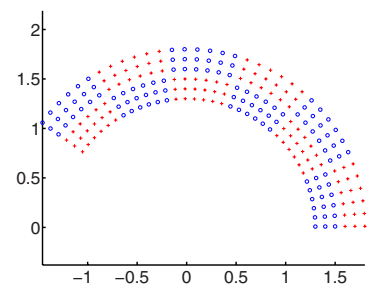

(e)

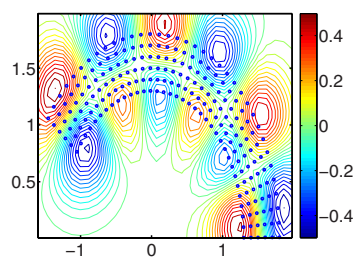

(f)

Fig. 3. (a) Samples with angle-based labels. (b) Contour plot for kFDA projection corresponding to (a). (c) Samples with radius-based labels. (d) Contour plot for kFDA projection corresponding to (c). (e) Samples with labels determined by both angle and radius. (f) Contour plot for kFDA projection corresponding to (e). (Blue labels marked 'o' indicate one class and red labels marked ' + ' indicate the other class in (a), (c) and (e). Colors refer to the online version of the paper.)

regions and alternately assumes positive and negative values as we move along the angular direction across these four regions. We may also observe that the seventh kPCA component (Fig. 2(i)) individually captures the radial change in the data and that it smoothly increases from negative to positive values as we step along the data in the radial direction.

kFDA projections: The previous synthesized dataset addressed the issue of estimating the probability density of these samples. We now reformulate this experiment as one requiring classification, i.e., as an experiment aiming to find the optimal separator between two groups of samples, as depicted by red and blue labels in Fig. [3(a), (c) and (e). We assigned labels in the angular direction to this dataset as shown in Fig. 3(a) and applied kFDA, which successfully discriminated between the two groups, as shown Fig. 3(b). (The kFDA projection in Fig. 3(b) roughly corresponds to the $5^{\text {th }} \mathrm{kPCA}$ component in Fig. 2(g).) We also assigned labels in the radial direction to this dataset as shown in Fig. [3(c) and applied kFDA, which again discriminated very well between the two groups, as seen in Fig. 3(d). (The kFDA projection in Fig. 3(d) roughly corresponds to the $7^{\text {th }} \mathrm{kPCA}$ component in Fig. 2(i).) We also conducted experiments where the labels were assigned to indicate changes in both the radial and angular directions and we have found that the kFDA projection (which did not correspond to any $\mathrm{kPCA}$ component) still separated the groups in an optimal manner (please see Fig. 3(e) and Fig. 3(f)). 


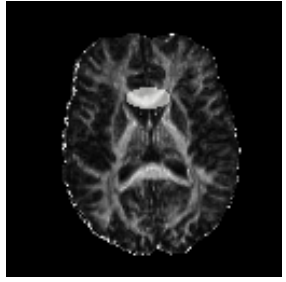

(a)

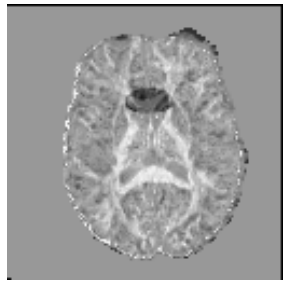

(b)

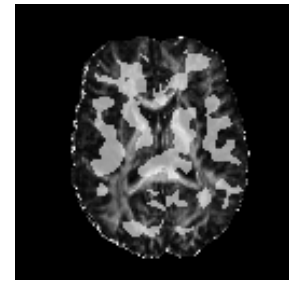

(a)

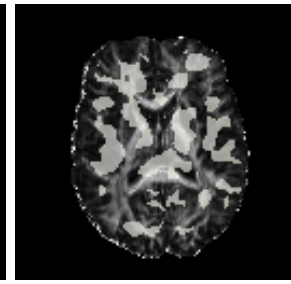

(b)
Fig. 4. (a) ROI with changes (highlighted) overlaid on the template FA map. (b) kFDA voxel-wise p-map (with significant low p-values highlighted) overlaid on the same FA map. Low pvalue regions in (b) match the true ROI in (a).
Fig. 5. (a) Schizophrenia kFDA p-map thresholded at 0.02 and overlaid on the template FA map. (b) Schizophrenia kFDA p-map thresholded using a false discovery rate of 0.18 and overlaid on the template FA map. Significant p-values after thresholding are highlighted in (a), (b).

Comparison to manifold learning: Manifold learning techniques such as ISOMAP (isometric mapping) [5] have also been proposed for DTI statistical analysis. We performed such an analysis using two approaches, ISOMAP [6], on the lines of [5], and MVU (maximum variance unfolding) [13], both of which correctly identify the underlying manifold of dimension 2 when applied to the dataset in Fig. 2(a), with MVU doing better than ISOMAP in separating the angular and radial changes in the data into its two components. An application of the Hotelling $T^{2}$ test on the MVU or ISOMAP embeddings, when group labels were assigned as in Figs. 3(a) or 3(c), yielded higher p-values, indicating less significant differences, than those found by applying a t-test on the kFDA projections or on the appropriate kPCA components, indicating that both MVU and ISOMAP were unable to identify the statistical distribution on the manifold like $\mathrm{kPCA}$ or determine projections like $\mathrm{kFDA}$ that separate the groups.

Having successfully validated our methods on a simulated non-imaging dataset with radial and angular variation, we shall now validate them on real DTI data with simulated changes in both the tensor eigenvalues and eigenvectors that emulate this joint radial and angular variation.

\subsection{Statistical Analysis of DTI Datasets with Simulated Changes}

Dataset Description: Our DTI dataset consisted of scans of 36 healthy volunteers (17 male and 19 female), acquired at dimensions $128 \times 128 \times 40$ and a voxel size of $1.72 \times 1.72 \times 3.0 \mathrm{~mm}$. These DT images were warped to a template, which was chosen as the scan of an additional healthy subject. We then identified an ROI on the template in the corpus callosum, as shown in Fig. 4(a), and introduced random changes in the principal eigenvalue and the azimuthal angle for the principal eigenvector of each tensor into the appropriate ROI for all unwarped subject DT images. The random changes were designed to slightly 
increase the principal eigenvalue and the principal azimuthal angle, on average, but were subtle enough so that these changes could not be visibly easily discerned on an FA map or a colormap for the principal direction. The DT images with the introduced random changes were then warped back to the template resulting in 36 DT images belonging to the abnormal class.

The simplest approach to forming the voxel-based sample vector is to use the 6 -independent components of the tensor at a voxel. However, we can potentially obtain more significant regional differences by incorporating neighborhood information around a voxel into our samples, in two different ways. In one approach, which we call the "long-vector" approach, we form a local spherical neighborhood around each voxel and combine the 6-independent components from each tensor in the local neighborhood into a long vector, which we use as our sample. In an alternative approach, we first smooth the DTI [14 data and then use the 6 -independent tensor components at a voxel as our sample. We shall now present results obtained by applying $\mathrm{kPCA}$ and $\mathrm{kFDA}$ to these different sample types.

Results Using kPCA, kFDA and Other Techniques: We have shown the central axial slice of the 3 -D p-value map found from an application of $\mathrm{kFDA}$ and the t-test in Figure 4 (b). We may observe that significant regions identified in Figure 4(b) match the true ROI very well. In Table 1, we have presented a detailed quantitative performance comparison of different approaches to discovering the true ROI. Our performance measure was the percentage overlap of voxels in the detected ROI (p-value map thresholded at a cut-off of 0.1 ) with the voxels in the true ROI. We have applied the different approaches to the original DTI dataset as well as to a smoothed version of the DT images, smoothed in the Log-Euclidean domain 14 using an isotropic truncated Gaussian filter with the filter $\sigma$ set to $4 \mathrm{~mm}$. This choice for smoothing yielded good results for the technique in 5. For the long-vector results, we used a spherical neighborhood of $4 \mathrm{~mm}$ radius that contained 39 voxels. The Gaussian RBF kernel width parameter $\sigma^{2}$ was set to $4 e-4$ in all kernel-based results not using the long-vector and this value was multiplied by the number of neighborhood voxels when the long-vector approach was used.

Rows 1 and 2 in Table 1 present results from conventional ADC and FA analyses and row 3 presents results using the ISOMAP technique from [5]. Since the $\mathrm{ADC}$ and FA concentrate on eigenvalue differences, they are not particularly sensitive in detecting the ROI. The ISOMAP technique (row 3) performs better than these two approaches, but it is hampered by the fact it does not use knowledge of the statistical distribution underlying the tensors. Knowledge of this statistical distribution led to enhanced results using the $\mathrm{kPCA}$ technique, as can be seen in rows 4-8. Rows 4 and 5 present results using the 6 independent tensor components as the sample vector, whereas rows 6-8 use the long-vector approach. We may observe that $\mathrm{kPCA}$, when used appropriately, is very sensitive in detecting the ROI. While the long-vector $\mathrm{kPCA}$ approach yielded poor results on the original DT data, it yielded good results on the smoothed DTI. From these kPCA results, we may also observe a general difficulty with using this method and the ISOMAP-based method, which stems from the fact that 
Table 1. Percentage overlap of detected ROI (p-value map thresholded at a cut-off of 0.1 ) with the true ROI (Please see text for explanation)

\begin{tabular}{|r|l|r|r|}
\hline & Analysis Method & Original DTI & Smooth DTI \\
\hline 1. & ADC & 14.92 & 21.71 \\
2. & FA & 39.14 & 42.39 \\
\hline 3. & ISOMAP- 3 components & 35.60 & 44.46 \\
\hline 4. & kPCA- 3 components & 60.41 & 72.53 \\
5. kPCA- 8 components & 54.95 & 62.48 \\
6. kPCA-long-vector 3 components & 13.44 & 53.32 \\
7. kPCA-long-vector 8 components & 44.46 & 75.18 \\
8. kPCA-long-vector 12 components & 51.70 & 81.83 \\
\hline 9. & kFDA & 57.90 & 71.64 \\
10. & kFDA-long-vector & $\mathbf{7 9 . 1 7}$ & $\mathbf{8 3 . 3 1}$ \\
11. kFDA-long-vector-cross-validated & 56.72 & 51.55 \\
\hline
\end{tabular}

we do not know the discriminatory component(s) in these representations, making it hard to select the component(s) on which the subsequent statistical tests must be applied. The kFDA approach (rows 9-11), on the other hand, does not suffer from this problem. As seen from row 10, kFDA employing the longvector approach on smooth DT images yields the best performance among all methods compared.

We note that kFDA is the only technique in Table 1 that uses the class labels while computing its features and hence it requires a special form of crossvalidation. We divided our data equally into training and testing samples. We then learned discriminatory directions on the training samples, used these directions to obtain projections for the testing samples and then applied the t-test on these test sample projections. Such a naive cross-validation approach would reduce performance considerably because the training is performed on half the number of samples and also partly because the p-values are computed from a fewer number of testing samples. However, row 11 in Table 1 indicates that the kFDA performance remains significantly better than several other approaches.

\subsection{Statistical Analysis of Schizophrenia DTI Dataset}

In addition to the 36 healthy volunteers used in Sec. 3.2, our dataset now additionally included scans of 34 schizophrenic patients (21 male and 13 female), again warped to the template. We first smoothed this dataset and applied kFDA with the 6 tensor components and the t-test voxel-wise (using the same smoothing and kernel parameters as in Sec. 3.2) to obtain a p-value map showing regional differences. The resulting significant regions, after thresholding the pvalue map at a cut-off of 0.02 and removing small spurious clusters, are shown in Fig. 5(a). Kernel FDA has found a number of white matter regions such as the corpus callosum and the internal capsule and gray matter regions such as some regions in the fronto-temporal cortex that have been implicated in other studies of schizophrenia. We plan to investigate the clinical significance of our 
findings in future work. (We note that we found similar significant regions when the p-value maps were alternatively thresholded using a false discovery rate [15] of 0.18 , as shown in Fig. $5(\mathrm{~b})$.)

\section{Conclusion}

Using both simulated and real data, we have established that kernel-based methods pave the way to resolving major issues in group-wise DTI statistical analysis, namely, the density estimation of voxel-based tensors (in the presence of nonlinearity), the appropriate representation of these tensors on a manifold and the subsequent use of this representation in the identification of features that can optimally identify region-based group differences. In particular, we have shown that kFDA can form the basis of a highly sensitive method for group-wise DTI statistical analysis. Such a method can open up numerous avenues of research in various DTI applications requiring large scale group analysis such as studies of various brain disorders involving prognosis, diagnosis or progression of disease. Future work will involve optimal kernel selection in kFDA [12] and more sophisticated cross-validation of our kFDA results using permutation tests on the DTI dataset with simulated changes as well as on the schizophrenia DTI dataset.

\section{Acknowledgements}

This work was supported by the National Institute of Health via grants R01MH070365, R01MH079938 and R01MH060722.

\section{References}

1. LeBihan, D., Mangin, J.F., et al.: Diffusion tensor imaging: Concepts and applications. J. of Magnetic Resonance Imaging 13, 534-546 (2001)

2. Wu, Y.C., Field, A.S., et al.: Quantitative analysis of diffusion tensor orientation: Theoretical framework. Magnetic Resonance in Medicine 52, 1146-1155 (2004)

3. Lenglet, C., Rousson, M., Deriche, R., Faugeras, O.: Statistics on the manifold of multivariate normal distributions: Theory and application to diffusion tensor MRI processing. Journal of Math. Imaging and Vision 25(3), 423-444 (2006)

4. Fletcher, P.T., Joshi, S.: Principal geodesic analysis on symmetric spaces: Statistics of diffusion tensors. In: Sonka, M., Kakadiaris, I.A., Kybic, J. (eds.) Computer Vision and Mathematical Methods in Medical and Biomedical Image Analysis. LNCS, vol. 3117, pp. 87-98. Springer, Heidelberg (2004)

5. Verma, R., Davatzikos, C.: Manifold based analysis of diffusion tensor images using isomaps. In: IEEE Int. Symp. on Biomed, Imaging, pp. 790-793. IEEE, Washington, DC, USA (2006)

6. Burges, C.: Geometric Methods for Feature Extraction and Dimensional Reduction. In: Data Mining and Knowledge Discovery Handbook, Kluwer Academic Publishers, Dordrecht (2005) 
7. Zhang, H., Yushkevich, P., et al.: Deformable registration of diffusion tensor MR images with explicit orientation optimization. Medical Image Analysis 10(5), 764785 (2006)

8. Cao, Y., Miller, M., Mori, S., Winslow, R., Younes, L.: Diffeomorphic matching of diffusion tensor images. In: CVPR-MMBIA, 67 (2006)

9. Xu, D., Mori, S., Shen, D., van Zijl, P., Davatzikos, C.: Spatial normalization of diffusion tensor fields. Magnetic Resonance in Medicine 50(1), 175-182 (2003)

10. Scholkopf, B., Smola, A.: Learning with Kernels. The MIT Press, Cambridge, MA (2002)

11. Girolami, M.: Orthogonal series density estimation and the kernel eigenvalue problem. Neural Computation 14(3), 669-688 (2002)

12. Kim, S.J., Magnani, A., Boyd, S.: Optimal kernel selection in kernel Fisher discriminant analysis. In: ACM Int. Conf. on Machine Learning 2006 (2006)

13. Saul, L.K., Weinberger, K.Q., Ham, J.H., Sha, F., Lee, D.D.: Spectral methods for dimensionality reduction. In: Semisupervised Learning, MIT Press, Cambridge, MA (2006)

14. Arsigny, V., Fillard, P., et al.: Medical Image Computing and Computer-Assisted Intervention. In: Duncan, J.S., Gerig, G. (eds.) MICCAI 2005. LNCS, vol. 3749, pp. 115-122. Springer, Heidelberg (2005)

15. Genovese, C., Lazar, N., Nichols, T.: Thresholding of statistical maps in functional neuroimaging using the false discovery rate. NeuroImage 15, 870-878 (2002) 